\title{
CALOGERO MODELS FOR DISTINGUISHABLE PARTICLES
}

\section{Cyril FURTLEHNER and Stéphane OUVRY 円}

Division de Physique Théorique 2, IPN, Orsay Fr-91406

\begin{abstract}
Motivated by topological bidimensional quantum models for distinguishable particles, and by Haldane's definition of mutual statistics for different species of particles, we propose a new class of one-dimensional $1 / r_{i j}^{2}$ Calogero model with coupling constants $g_{i j}$ depending on the labels of the particles. We solve the groundstate problem, and show how to build some classes of excited states.
\end{abstract}

IPNO/TH 94-02

May 1994

\footnotetext{
${ }^{1}$ and LPTPE, Tour 16, Université Paris 6/ electronic e-mail: OUVRY@FRCPN11

${ }^{2}$ Unité de Recherche des Universités Paris 11 et Paris 6 associée au CNRS
} 


\section{INTRODUCTION}

It has been known for some time [1] that there is a correspondance between the $1 / r^{2}$ $N$-body Calogero model [2] in an harmonic well and the $N$-anyon model [3] projected in the lowest Landau level of an external magnetic field. This correspondance can be seen at the level of the spectrum -an explicit mapping between both models is however still missing-, and also at the level of thermodynamical quantities, such as the equation of state of both systems [4], [5]. Actually, one finds thermodynamical quantities [6] appropriate for describing a system of particles with fractional statistics à la Haldane [7]. This last result suggests in particular that Haldane's statistics is essentially a one dimensional projection of the anyonic statistics, which is a bidimensional concept.

A point of particular interest in Haldane's framework is the possibility to have particles of different species, with mutual statistical coupling parameter depending on the species coupled. This is to be related to the generalisation of the anyon model to a model of bidimensional distinguishable particles [8], with mutual topological interactions (and possibly an external magnetic field). Here, one starts from a generalized Chern-Simons Lagrangian with $N$ independant gauge fields coupled to $N$ particles via an $N \mathrm{x} N$ coupling matrix. This model is truly a "toplogical quantum model", which narrows down to the usual anyon model if one insists on particles indistinguishability and on related statistical considerations.

The existence of such models for different species of particles strongly suggests, through a line of reasonning identical to the one used above, the possibility of generalising the Calogero model to a model of distinguishable particles with $1 / r_{i j}^{2}$ couplings depending on the labelling of the particles $\{i, j\}$ coupled. It might then happen that some sort of mapping exists between this new type of one-dimensional models and the topological bidimensional quantum model in a strong magnetic field. 
In this short note we propose a possible definition of such a generalisation of the Calogero model. Far from being able to find the desired mapping, if any, we simply exhibit the groundstate and show how to find some classes of excited states. The problem of the integrability of this new model remains entire.

\section{THE CALOGERO MODEL : A REMINDER}

The usual Calogero model [2] is defined by the Hamiltonian

$$
H=\frac{1}{2}\left[\sum_{i=1}^{N}-d_{i}{ }^{2}+x_{i}{ }^{2}+\sum_{j \neq i} \frac{g}{x_{i j}{ }^{2}}\right]
$$

where one has considered particles of unit mass in an harmonic well of unit strength. The eigenstates must have a well-defined asymptotic behavior to have an Hilbert space stable under the action of the Hamiltonian

$$
\psi \sim_{x_{i j}=0} x_{i j}^{\nu}
$$

with $\nu$ solution of $g=\nu(\nu-1)$.

Since one wants a well defined groundstate, $g$ has to be greater than $-1 / 4$. Following standard arguments [2], one specifies

$$
\nu=\frac{1}{2}+\frac{\sqrt{1+4 g}}{2}
$$

From this particular asymptotic behavior ( $\nu$ positive), one concludes that the particles are umpenetrable (the current also vanishes at coinciding points).

To proceed further one can perform [1] on the Hilbert space the non unitarity transformation

$$
\psi=\prod_{j<i}\left|x_{i}-x_{j}\right|^{\nu} \tilde{\psi}
$$


This transformation explicitely encodes the asymptotic behaviour given in eq.(2). One gets a transformed Calogero Hamiltonian

$$
\tilde{H}=\frac{1}{2}\left[\sum_{i=1}^{N}-d_{i}^{2}+x_{i}^{2}-\sum_{j \neq i} \frac{\nu}{x_{i j}}\left(d_{i}-d_{j}\right)\right]
$$

Calogero [2] has shown that eigenstates of this Hamiltonian have to be completely symetric.

The very fact that a particular asymptotic behaviour is imposed on the eigenstates at coinciding points implies that different particles configurations are disconnected. This means that the Hamiltonian is still hermitian if restricted on a Hilbert space corresponding to a given particle configuration, i.e. to a given ordering of particles (in other words the particles are unpenetrable). Thus, an eigenstate on a given configuration can be used to define an eigenstate on a different configuration by simply multiplying the eigenstate by a constant which depends on the desired statistics

$$
\psi(P x)=\epsilon_{P} \psi(x)
$$

where $\mathrm{P}$ is the permutation between both configurations. For example, $\epsilon_{P}=1$ for a bosonic statistics, and for a fermionic statistics, $\epsilon_{P}$ is the sign of the permutation. It is however clear that the information is entirely contained in a given configuration.

Now, if one decides to restrict oneself to the Hilbert space of totally symetric eigenstates, the transformed Calogero Hamiltonian coincides with [1]

$$
\tilde{H}=\frac{1}{2} \sum_{i=1}^{N}\left(a_{i}^{-} a_{i}^{+}+a_{i}^{+} a_{i}^{-}\right)
$$

where the creation and annihilation operators have been defined as

$$
a_{i}^{ \pm}=\frac{1}{\sqrt{2}}\left(x_{i} \mp D_{i}\right)
$$

with

$$
D_{i}=d_{i}+\sum_{j \neq i} \frac{\nu}{x_{i j}}\left(1-K_{i j}\right)
$$


The commutation rules are

$$
\begin{gathered}
{\left[D_{i}, x_{j}\right]=\delta_{i j}\left(1+\sum_{k=1}^{N} \nu K_{i k}\right)-\nu K_{i j}} \\
{\left[D_{i}, D_{j}\right]=0} \\
{\left[a_{i}^{ \pm}, a_{j}^{ \pm}\right]=0} \\
{\left[a_{i}^{-}, a_{j}^{+}\right]=\delta_{i j}\left(1+\sum_{k=1}^{N} \nu K_{i k}\right)-\nu K_{i j}} \\
{\left[\tilde{H}, a_{i}^{ \pm n}\right]= \pm n a_{i}^{ \pm}}
\end{gathered}
$$

This algebra entirely describes the Calogero model.

As a remark, if one had rather decided to use antisymetric eigenstates, one would have defined

$$
D_{i}=d_{i}+\sum_{j \neq} \frac{\nu}{x_{i j}}\left(1+K_{i j}\right)
$$

and have applied the same procedure but now on a totally antisymetric space.

Or, following [1], one could as well have redefined differently the eigenstates

$$
\psi=\prod_{j<i}\left|x_{i}-x_{j}\right|^{-\nu} \tilde{\psi}
$$

and then constructed the same algebra as above but with

$$
D_{i}=d_{i}-\sum_{j \neq i} \frac{\nu}{x_{i j}}\left(1-K_{i j}\right)
$$

(and built the eigenstate with the new creation operators on the antisymetric subspace). In any case, one stresses again that all the information is contained in the totally symetric space.

\section{GENERALISATION TO A SYSTEM OF NON IDENTICAL PARTICLES}


What has just been said above can be used to generalise the Calogero model to a system of non identical particles. The main ingredients or building blocks of the construction should still be the asymptotic behavior at coinciding points, and the algebra of creationannihilation operators once this asymptotic behavior has been extracted. However, in a situation where the particles are no more identical, once a configuration is given, there is obviously no way to restrict oneself to totally symetric eigenstates.

We define the Hamiltonian

$$
H=\frac{1}{2}\left[\sum_{i=1}^{N}-d_{i}^{2}+x_{i}^{2}+\sum_{j \neq i} \frac{g_{i j}}{x_{i j}^{2}}+\sum_{i, j, k \neq} \frac{\nu_{i j} \nu_{i k}}{x_{i j} x_{i k}}\right]
$$

with $g_{i j}=\nu_{i j}\left(\nu_{i j}-1\right)$ (again the $g_{i j}$ 's have to be greater than $\left.-1 / 4\right)$. One remarks that

i) when all the $\nu_{i j}$ 's are equal, this model narrows down to the usual Calogero model (1)

ii) the occurence of 3-body interactions is quite reminiscent of what happens in the anyon model [3]

iii) the eigenstates have the same type of asymptotic behavior as in the usual Calogero model $\psi \sim_{x_{i j}=0} x_{i j}^{\nu_{i j}}$ with $\nu_{i j}=\frac{1}{2}+\frac{\sqrt{1+4 g_{i j}}}{2}$.

The non unitary transformation

$$
\psi=\prod_{j<i} x_{i j}^{\nu_{i j}} \tilde{\psi}
$$

leads to the Hamiltonian

$$
\tilde{H}=\frac{1}{2}\left[\sum_{i=1}^{N}-d_{i}^{2}+x_{i}^{2}-\sum_{j \neq i} \frac{\nu_{i j}}{x_{i j}}\left(d_{i}-d_{j}\right)\right]
$$

where the 2 an 3-body interactions have simply disappeared. Again, this is quite reminiscent of the anyon model where the 3-body interactions can be erased, altogether with the singular 2-body interactions (singular in perturbation theory), by the non unitary transformation $\prod_{i<j} r_{i j}^{|\alpha|}$, where $\alpha$ is the statistical parameter [9]. 
The just obtained non hermitian Hamiltonian is quite analogous to the one encountered in the standard Calogero case. Following the same procedure as above, we define the covariant derivative

$$
D_{i}=d_{i}+\sum_{j \neq i} \frac{\nu_{i j}}{x_{i j}}\left(1-K_{i j}\right)
$$

Its commutator with $x_{i}$ becomes

$$
\left[D_{i}, x_{j}\right]=\delta_{i j}\left(1+\sum_{k=1}^{N} \nu_{i k} K_{i k}\right)-\nu_{i j} K_{i j}
$$

The main difference is that $\left[D_{i}, D_{j}\right]$ is now nontrivial but happens to vanish on totally symetric eigenstates. Thus, one can easily construct some classes of eigenstates in this particular space. Indeed, for totally symetric functions, the Hamiltonian can be written as

$$
\tilde{H}=\frac{1}{2} \sum_{i=1}^{N}\left(a_{i}^{-} a_{i}^{+}+a_{i}^{+} a_{i}^{-}\right)
$$

with the same definition for the $a_{i}$ 's as above. On this symmetric space, the commutation relations reduce to

$$
\begin{gathered}
{\left[a_{i}^{ \pm}, a_{j}^{ \pm}\right]=0} \\
{\left[a_{i}^{-} a_{j}^{+}\right]=\delta_{i j}\left(1+\sum_{k=1}^{N} \nu_{i k}\right)-\nu_{i j}}
\end{gathered}
$$

The solution of

$$
a_{i}^{-} \tilde{\psi}=0
$$

is $\tilde{\psi}_{0}=\exp \left(-\sum_{i=0}^{N} \frac{x_{i}^{2}}{2}\right)$. It is the groundstate since it has has no node. Its energy is simply $E_{0}=\frac{N}{2}+\frac{1}{2} \sum_{i \neq j} \nu_{i j}$.

In analogy with the standard Calogero system, we might also consider the eigenstates

$$
\tilde{\psi}_{n}=\left(\sum_{i=0}^{N} a_{i}^{+}\right)^{n} \tilde{\psi}_{0}
$$


The relation $a_{i}^{-} \tilde{\psi}_{n}=n \tilde{\psi}_{n-1}$ makes it easy to see that $\tilde{H} \tilde{\psi}_{n}=\left(E_{0}+n\right) \tilde{\psi}_{n}$, i.e. $\tilde{\psi}_{n}$ is an eigenstate of $\tilde{H}$ with energy $E_{0}+n$. Another directly generalised eigenstate of the Calogero model is

$$
\tilde{\psi}_{21}=\left(\sum_{i=0}^{N}{a_{i}^{+2}}^{2}\right) \tilde{\psi}_{0}=\sum_{i=1}^{N}\left[2 x_{i}{ }^{2}-\left(1+\sum_{l \neq i} \nu_{i l}\right)\right] \tilde{\psi}_{0}
$$

with $E_{0}+2$ for eigenvalue. Actually it is easy to see that any eigenstate of the form

$$
\tilde{\psi}(x)=P_{n}(x) \tilde{\psi}_{0}(x)
$$

with $P_{n}$ a polynomial of degree $n$ in the variables $x_{i}$ has necesseraly $E_{0}+n$ for eigenvalue since

$$
\tilde{H} \tilde{\psi}(x)=\left[\left(E_{0}+n\right) P_{n}(x)+Q_{n-2}(x)\right] \tilde{\psi}_{0}(x) .
$$

Clearly, $Q_{n-2}$, a polynomial of degree $n-2$, has to vanish for $\tilde{\psi}$ to be an eigenstate of energy $E_{0}+n$.

We could go on and consider for example the state

$$
\tilde{\psi}_{31}=\left(\sum_{i=0}^{N} a_{i}^{+3}\right) \tilde{\psi}_{0}=\sqrt{2} \sum_{i=1}^{N}\left[2 x_{i}{ }^{3}-3 x_{i}\left(1+\sum_{l \neq i} \nu_{i l}\right)\right] \tilde{\psi}_{0}
$$

However, it is not an eigenstate in the present case since

$$
\tilde{H} \tilde{\psi}_{31}=\left(E_{0}+3\right) \tilde{\psi}_{31}+\frac{3}{\sqrt{2}} \sum_{j \neq i} \frac{\nu_{i j}}{x_{i j}}\left(\sum_{l \neq i} \nu_{i l}-\sum_{l \neq j} \nu_{j l}\right) \tilde{\psi}_{0}
$$

Clearly, the last term in the right hand side of (32) vanishes if and only if all the $\nu_{i j}$ 's are equal.

To close this analysis, let us consider the subspace of the totally symetric space stable under the action of $\tilde{H}$. It is defined by

$$
0=\sum_{j \neq k, k+1}\left(\nu_{j k}-\nu_{j k+1}\right)\left(\frac{1}{x_{j k}}\left(d_{j}-d_{k}\right)-\frac{1}{x_{j k+1}}\left(d_{j}-d_{k+1}\right)\right)
$$


for $k=1, \ldots, N-1$. On this particular subspace $\tilde{H}$ coincides with the usual Calogero Hamiltonian with $\nu=\frac{2}{N(N-1)} \sum_{i \neq j} \nu_{i j}$. Thus, projecting the Calogero eigenstates on this subspace, gives all the eigenstates of the model in this subspace. But it certainly does not provide the entire set of eigenstates, simply because most of the eigenstates do not have a symetrical analytic form, as already stressed above. This is obviously due to $\tilde{H}$ not being anymore symetrical, contrary to what happens in the standard Calogero case.

\section{CONCLUSION}

In conclusion, a generalisation of the Calogero model has been proposed which presents some analogy with the way the anyon model is extended to a general topological quantum model [8]. The similarities are patent when the asymptotic behavior at coinciding points, and the analytical form of the ground state and of some excited states, are considered. Unfortunately, the lack of an explicit mapping between the $N$-body Calogero model and the $N$-anyon model in the lowest Landau level (only in the 2-body case one has been able to construct an explicit mapping [10]) did not allow for a direct proof of this correspondance, and thus for a systematic way to build the entire spectrum. On the other hand, the algebraic approch displayed in [1] has been useful to construct some excited states. It would certainly be quite interesting to find a way towards a complete resolution of this multi-species Calogero model. 


\section{REFERENCES :}

1) L. Brink, T.H. Hansson, S. Konstein and M.A. Vasiliev, Nucl. Phys. B401 (1993) 591 ; T.H. Hansson, J. M. Leinaas and J. Myrheim, Nucl. Phys. B384 (1992) 559 ; A. P. Polychronakos, Phys. Rev. Lett. 69 (1992) 7703

2) F. Calogero, J. Math. Phys. 10 (1969) 2191, 2197 ; 12 (1971) 419

3) J. M. Leinaas and J. Myrheim, Nuovo Cimento B37 (1977) 1

4) A. Dasnieres de Veigy and S. Ouvry, Phys. Rev. Lett. 72 (1994) 600

5) S. Isakov, Int. Jour. Mod. Phys. A9 (1994) 2563; Int. Jour. Mod. Phys. B8 (1994) 319

6) Y-S Wu, Utah Preprint (1994); D. Bernard and Y-S Wu, Saclay Preprint (1994); M.V.N. Murthy and R. Shankar, IMSc Preprint (1994)

7) F. D. M. Haldane, Phys. Rev. Lett. 67 (1991) 937

8) A. Dasnieres de Veigy and S. Ouvry, Phys. Lett. B307 (1993) 91

9) A. Comtet, J. McCabe and S. Ouvry, Phys. Lett. B260 (1991) 372

10) J.M. Leinaas, private communication. 